\title{
Three-dimensional model of the Rzeczka object (the Riese Complex, Sowie Range) as an enhancement of the comprehensive geotourism potential of an underground tourist route
}

\author{
Katarzyna Kądziołka ${ }^{1}$, Damian Kasza ${ }^{1, *}$, and Wojciech J. Milczarek ${ }^{1}$ \\ ${ }^{1}$ Wrocław University of Science and Technology, Faculty of Geoengineering, Mining and Geology, 27 Wyb. Wyspiańskiego St., 50- \\ 370 Wrocław, Poland
}

\begin{abstract}
The dynamic development of measurement technology based on the use of laser scanners allows for fast transfer of information, related to terrestrial data, to a virtual environment and enables the creation of three-dimensional models. Because of the growing demand for underground areas inventory, especially historic areas, the creation of 3D numerical models increases in popularity. It allows to increase the geotouristic potential of a particular area and to use solutions in support of the safety of tourists as well as the safety of area itself. The development of the terrestrial laser scanning technique is accompanied simultaneously by the development of means of digital data processing that was obtained in the form of point clouds. Those means use the commercial software as well as software available in the open source system. Both computing environments use algorithms enabling the processing of huge collections of points in automatized mode by using a number of open-access algorithms either freeware or dedicated. The methodology of numerical models development, described in this article, has been presented on the example of underground object "Rzeczka" ("Riese" Complex) located at the Sowie Range (SW Poland).
\end{abstract}

\section{Introduction}

Currently, the terrestrial laser scanning is the fastest growing technique used for obtaining terrestrial data for closed areas. The advantages of this technique include high-speed data acquisition, high precision of measurement, obtaining information about the physical characteristics of examined/measured areas and the independence from lighting conditions, among many others [1].

The emitter of laser beam built into the scanner (own source of light) makes this measuring instrument perfect for underground areas inventory. However, the measurement itself is not all. Data processing and creation of numerical models are significant problems. Currently, algorithms used for the reconstruction of a surface out of point clouds allow for working with multimillion collections of data with a different degree of accuracy. The access to these collections can be obtained from the level of open source as well as commercial type of software.

In the era of growing popularity of underground complexes as geotouristic areas, the promotion of this type of areas becomes very important. The numerical three-dimensional models contain information about the present state of the area and can easily be used as an advertised product in the form of interactive visualizations and animations.

\section{Study area}

"Rzeczka" is one of seven currently discovered underground objects of a secret Nazi miningconstruction project done under the cryptonym "Riese". The project envisaged building a complex of interconnected underground excavations and ground infrastructure which most likely were supposed to function as factories and laboratories [2,3].

A complex of underground tunnels of "Rzeczka" has been excavated in the rock massif of Ostra Mountain in the Sowie Range (Lower Silesia, SW Poland). Three adits leading into the area are located at the East Mountainside of the above-named massif at the heights from 557 to 559 meters above sea level [2]. The available tourist route incorporates the whore area of underground excavations and is about $500 \mathrm{~m}$ in length whereas area's cubature is $14000 \mathrm{~m}^{3}$ (Fig. 1).

* Corresponding author: damian.kasza@pwr.edu.pl 


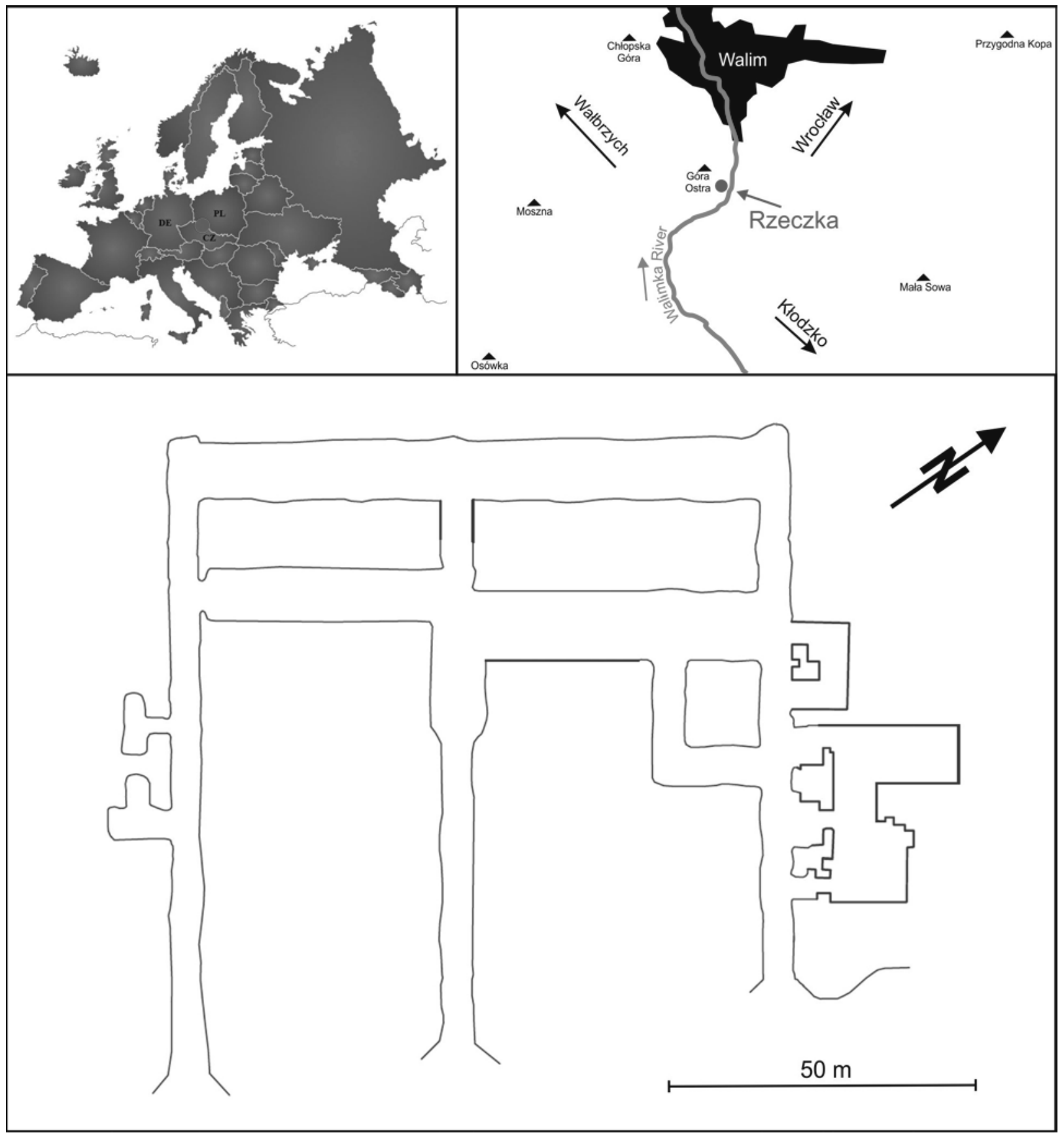

Fig. 1. Localization of "Rzeczka" underground complex and the area's plan.

\section{Terrestrial laser scanning technology}

Terrestrial laser scanning, also known as LiDAR (Light Detection and Ranging), is an active remote sensing system which uses instruments that emit electromagnetic waves in a form of the laser beam. Scanning is relatively new and ever-improving technique that involves collecting terrestrial data of examined areas and obtaining the location of these areas (points) in terms of the scanner. It allows for the fast acquirement of dense point models, in which every point is assigned not only to $x y z$ coordinates but also to an additional parameter which marks an intensity value of reflected wave [1].

The allocation of coordinates $x y z$ of a particular measurement point in the scanner's local system is executed by measuring the distance, vertical angle and horizontal direction. Basing on the knowledge of distances and directions of the point, the coordinate increments of the particular point are determined in terms of scanner's location and elevation (Fig. 2):

$$
\begin{aligned}
& \Delta x=s \times \cos \alpha \\
& \Delta y=s \times \sin \alpha \\
& \Delta z=s \times \cos \beta
\end{aligned}
$$

where $s$ denotes the distance between the scanned point and scanner, $\alpha$ denotes the value of the horizontal angle, $\beta$ denotes the vertical angle. 


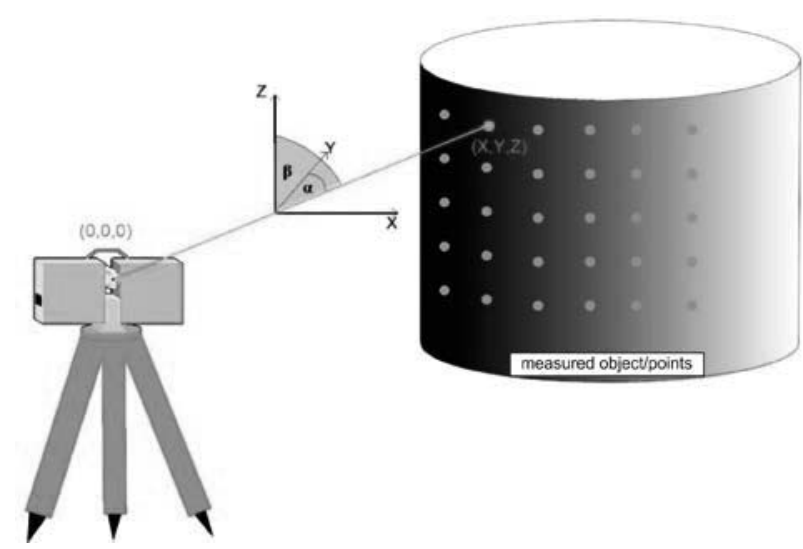

Fig. 2. The graphical representation of the essence of measurement taken by the laser scanner [4].

During the inventory works in "Rzeczka", the Leica's ScanStation C10 scanner has been used. Measurement stations were positioned not more than 5 meters away from each other in order to assure covering the whole area. The scanning resolution was $5 \times 5 \mathrm{~cm}$ per 50 meters of the distance. The HDS targets with a diameter of 6" were used as the reference points inside the underground excavations to connect individual measurement stations of the scanner. The final point cloud, created during the post-processing in the Leica Cyclone environment, has been linked to coordinate system PL2000 zone 7 by using 3 points which were marked in front of adits and measured with the use of RTK GPS technique.

\section{The analysis of the results}

The obtained data set consisted of nearly 120 million points. The point cloud was pre-treated in order to eliminate noise - incorrectly measured points. Some of it (that represented scanned operators' silhouettes, support stands with reference targets, etc.), has been removed manually. After that, the whole point cloud was filtrated by using the Noise filter tool, available in the Cloud Compare environment [5]. Ultimately - for more effective work related to mesh type surface reconstruction, the cloud was simplificated by the use of Poisson-disk sampling tool available in MeshLab application program [6]. This operation allowed for the unification of set by determining the minimal distance between points within the given value of $10 \mathrm{~cm}$. Thereby, the point cloud, after the pre-processing phase, consisted of nearly 14 million points and was prepared for the second phase of works - the creation of mesh type surface structure with the use of open source type of software - MeshLab and commercial type of software SketchUp.

\subsection{Surface reconstruction of mesh type in MeshLab application program}

MeshLab's environment has a few built-in algorithms allowing for generation of mesh type structure model based on data derived from laser scanning. For conducting a series of tests, the authors of this paper decided on picking 3 algorithms: Marching Cubes, Ball Pivoting and Poisson Surface Reconstruction.

\subsubsection{Marching Cubes}

This algorithm was designed by Lorensen and Cline in 1987 [7]. It works by creating a remote scalar field of a triangle mesh that approximates the equipotential surface. The surface is created by connecting 26 points that have equal value. In the Marching Cubes algorithm the scalar fields, built out of eight vertices that create a cube, are being analysed (Fig. 3) [8]. Each vertex is assigned with a value of 0 (when a vertex is located outside of the constructed surface) or 1 (when a vertex is located inside or on the wall of the presented surface).

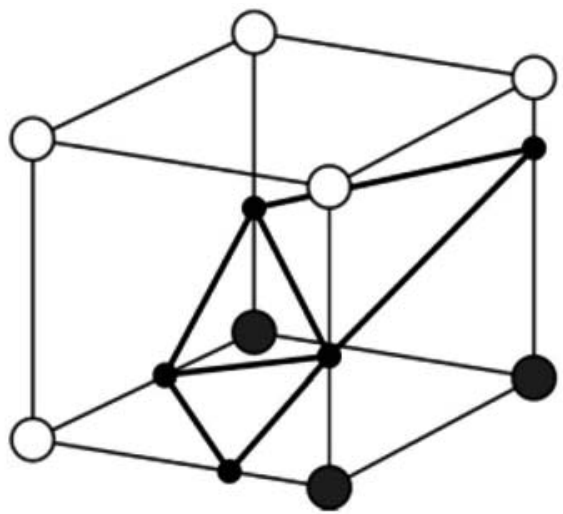

value equal 1

\section{value equal 0}

\section{vertex of generated triangles}

Fig. 3. The graphical representation of a structure of triangle mesh with the use of the Marching Cubes algorithm [8].

The process of generating the grid of triangles is based on counting up all the vertices placed on the edges of constructed cubes and connecting their intersections with cubes' surfaces to create triangles. The resolution of generated mesh, by the Marching Cubes algorithm, can be regulated by setting the number of cubes. The bigger the number of cubes, the smaller the dimension of each cube and consequently the greater accuracy of the model's projection (more details are included). However, each increase in number of cubes increases the time required for generating the triangle mesh that represents the model's surface [7].

\subsubsection{Ball Pivoting}

The creation of triangle mesh, with the use of Ball Pivoting algorithm, starts with determining the radius of the sphere that rotates around all the edges of created triangles. The moment when the sphere touches another point, which is contained in the point cloud, begins the appointing of a new triangle, made out of two points of the previous triangle that the sphere rotated around, and 
appointing of the third point which has just been encountered by the sphere. In this way, consecutive triangles come into being and create triangle mesh of the model. The rotating sphere generates further parts of the triangle mesh as long as its surface detects other points of the cloud (Fig. 4). In less frequent positioning of points or in areas of insuficient data, the generated model will have multiple gaps that entail an inaccurate projection of the area. The algorithm is suitable mainly for densely and evenly distributed points [9].

The precision of area's projection with the use of Ball Pivoting method depends on accurate choosing of the rotating sphere's radius. If the value of the radius is too small, there is a possibility of skipping many details as well as creating multiple gaps in the model [9].
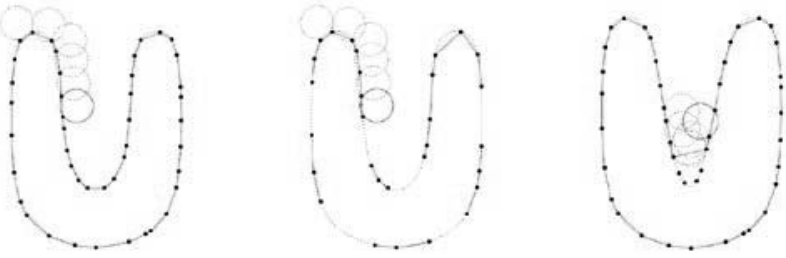

Fig. 4. The graphical representation of a structure of triangle mesh with the use of Ball Pivoting algorithm [9].

\subsubsection{Poisson Surface Reconstruction algorithm}

The Poisson Surface Reconstruction algorithm was designed by Kazhdan, Bolitho and Hoppe [10-13]. Oftentimes, this algorithm is implemented in programs that are used for developing point clouds. It is used particularly during the process of automatized creation of irregular triangle mesh TIN. The Poisson Surface Reconstruction algorithm is one of the most frequently applied one, among those that are being used for creating surfaces out of large collections of points, due to it's operating mode - it is a global method that employes the whole data set during the computational process without dividing it into smaller sample points for the purpose of approximating the modeled surface. According to this fact, the algorithm is immune to undesirable data distortion (e.g. gaps in point clouds).

The algorithm's work is based on the solution of the Poisson's equation. For the purpose of application of this algorithm, it is required beforehand to calculate normal vectors of all points of the formulated set. The first stage of the algorithm's work is to define the gradient of the particular set function and to determine its' simplified characteristic function. The next stage consists in approximating the gradient of the scalar field. For optimal alignment of the data set, the aligning filter based on Gauss equation is applied. The appointed in this way irregular triangle mesh TIN, which forms the surface of the modeled area, has the following features:

- huge detailedness of the projection of model's edges

- high immunity to the formation of distortion

- during the computational process an octree method is employed (lower computational resources load)
- a complete, so-called "closure" of the model (leaves no 'holes' in the model) [10].

\subsection{Surface reconstruction of mesh type in SketchUp application program}

The SketchUp software is a commercial environment which allows for 3D modeling, point clouds servicing, modeling buildings and other engineering structures. The software extended with the Undet plug-in allows for importing the point cloud. It also contains many tools allowing for efficient management and visualization of data. Undet enables generating and editing the mesh type surfaces. The Undet 2017 version of the program has an option for automatized detection of surfaces and construction of a grid of irregular triangles on the pointed area - it allows for determining mesh's parameters: size and curvature tolerance [14].

\section{Results and discussion}

The conducted tests for generating surface's structure of mesh type, for the chosen fragment of excavation by using 3 different algorithms available in the MeshLab software, brought diverse results. The Marching Cubes, as well as the Ball Pivoting algorithm, projected the model of underground excavations in an unsatisfactory degree. The model had multiple gaps (so-called holes, outgrowth, and other artifacts; Fig. 5A, B). In the microscopic evaluation of created models, the most detailed model has been generated by the Poisson Surface Reconstruction algorithm (Fig. 5C).

The test results presented in Fig. 5 made the choice clear and the Poisson Surface Reconstruction (PSR) algorithm has been used for constructing the model of "Rzeczka". In the first step, for prepared data set in the point cloud form (cf. Section 4.), normal vectors, which are necessary for the algorithm's work, were generated. Unfortunately, scanner's and software's producer does not provide the option for exporting information about normal vectors directly along with the collection of points. Normal vectors were generated with the use of compute normals for point sets tool. The process of calculating these vectors consists in determining the approximate local surface by using the location of points and their nearest 'neighbours'. The normal, in the specified point, vectors are perpendicular to the specified surface whereas the surface is set by the estimated triangulation method with the use of neighbouring points [6]. 


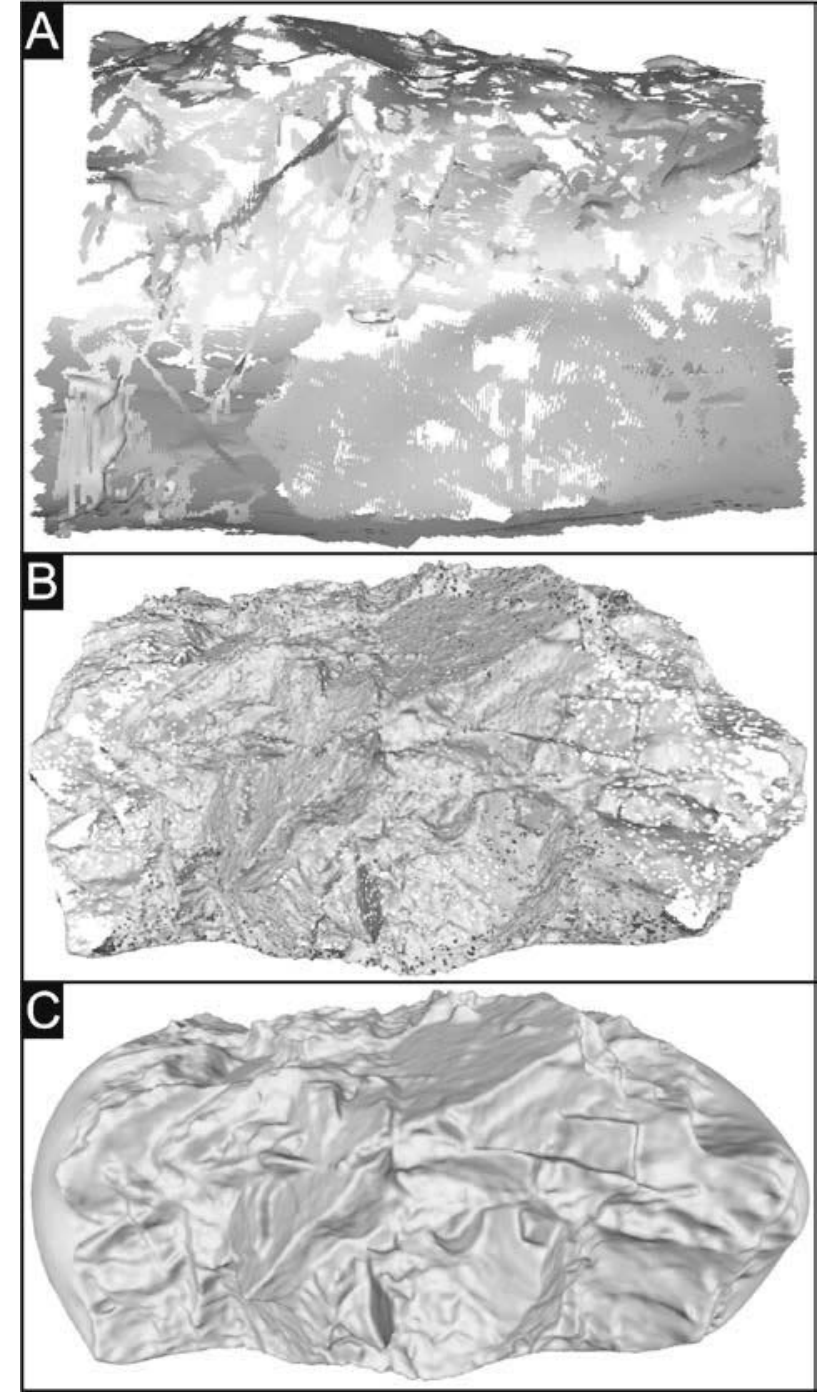

Fig. 5. The test results for mesh type surface creation with the use of Marching Cubes (A), Ball Pivoting (B) and Poisson Surface Reconstruction (C) algorithms.

The second step was the beginning of testing the parameters that create triangle mesh - mesh type structure with the use of the PSR algorithm. During the process, vertices, edges and surfaces representing the model are generated. These features are tangential only along the edges [15]. The most important parameter, which is responsible for the accuracy of area's projection, is the depth parameter (octree depth). Its' value determines the complexity of generated mesh and consequently the detailedness of the model. This parameter has a great influence on the run-time of the computational process. The higher its' value, the more time-consuming computational process. For the purposes of comparison, the model was generated with the depth being set to 8 (Fig. 6A) and 15 (Fig. 6B). The number of generated triangles depending on the parameter's value was presented in Table 1.
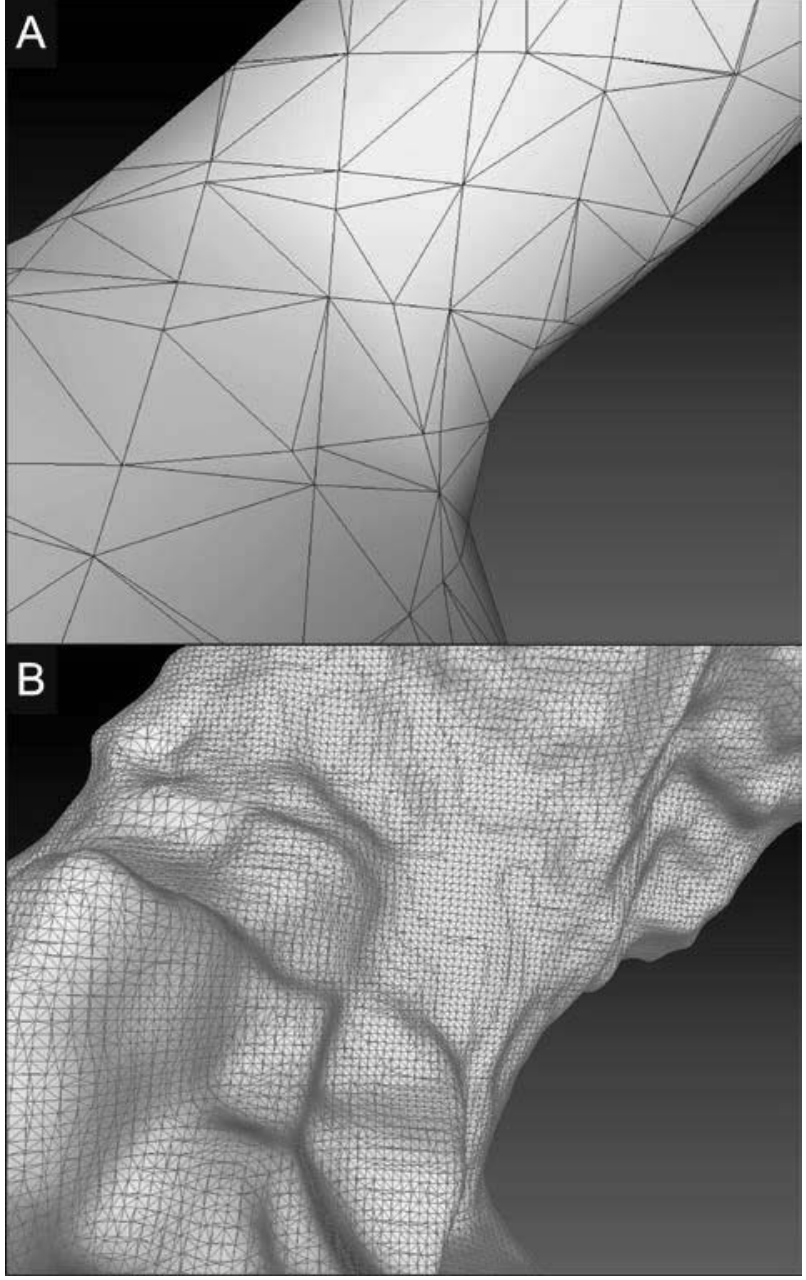

Fig. 6. A fragment of the model generated with the use of Poisson Surface Reconstruction algorithm: depth parameter set to $8(\mathrm{~A})$ and $15(\mathrm{~B})$.

Table 1. The comparison of the number of generated triangles depending on the value of depth parameter.

\begin{tabular}{|c|c|}
\hline Octree depth & Number of triangles \\
\hline 8 & 60568 \\
\hline 15 & 17103642 \\
\hline
\end{tabular}

Based on the presented comparison of generated models, one may conclude that there is a major difference in the accuracy of projecting model's details. The model with depth value set to 8 is clearly less accurate. Sharp edges of the underground excavation, which has complex geometry, were overly smoothed, therefore, the model does not represent the actual image of the area. The model's triangle mesh with depth value set to 15 is significantly more complex what makes the projection of the geometry of excavations more accurate. The details of the projected surface and its' sharp edges were preserved. The authors did not conclude the enhancement of model's detailedness along with a further increase in depth parameter's value. The final model is presented in Fig. 7. 


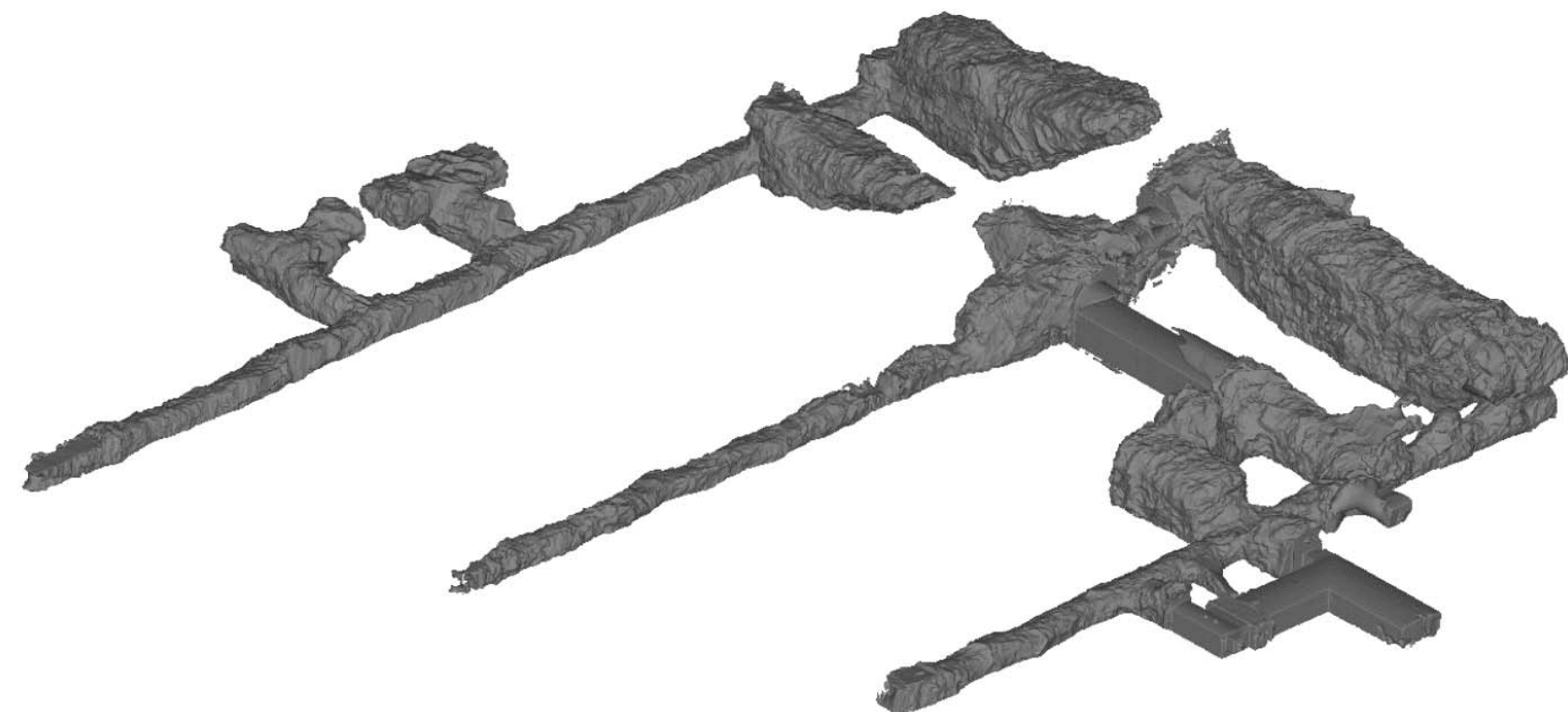

Fig. 7. The numerical model of the underground excavations of "Rzeczka" built in the MeshLab environment.

The three-dimensional model of "Rzeczka", in SketchUp's environment, was developed in Undet extension with the use of Feature extraction tool. In accordance with the description [14], this tool is suitable for all types of surfaces - buildings, engineering structures, roads, ground's surfaces, tunnels or caverns. In the presented case, this option generates surfaces in a specified (given) area of point clouds in a highly inaccurate manner, probably due to the complex geometry of the excavation (Fig. 8A). In the case of enclosed elements of the area, e.g. guardroom (simple geometric shapes), the surfaces were adequately designated and consequently the generated TIN mesh accurately represents surfaces/planes of these rooms (Fig. 8B).

The authors concluded that the point clouds conversion of the scanned area, which has complex geometry, into the mesh of irregular triangles done in the Undet for SketchUp program, requires constant evaluation of correctness on the user's part, i.e. determining the placement of the surface that was created with the use of Feature Extraction option, creating the mesh surface with the use of Convert to Mesh option and manual editing of the mesh: deletion of mistakenly generated triangles or change of surface's orientation that was proposed in the program. During the transformation of the designated area into the mesh structure, it is necessary to set the parameter of surface's curvature tolerance to maximum - in that, it is possible to project irregular and complex geometry of excavations, and to generate the numerical model (Fig. 9).

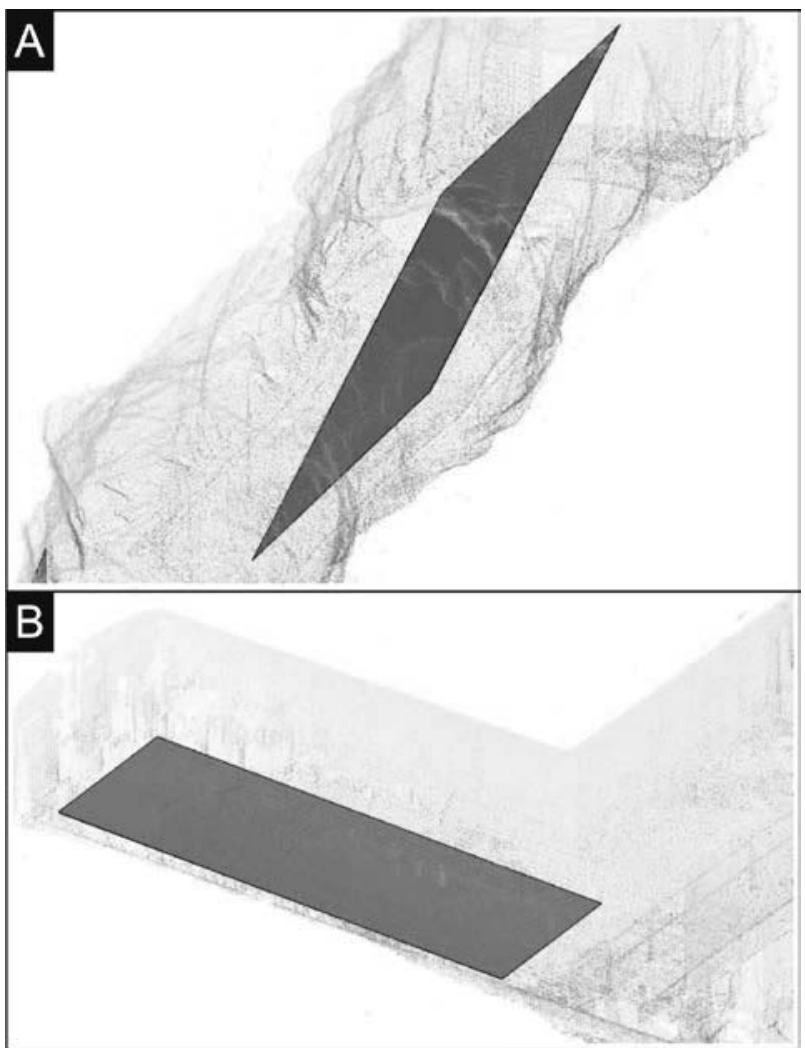

Fig. 8. Example of employing Feature extraction tool: incorrectly designated surface (A) and correctly designated surface (B). 


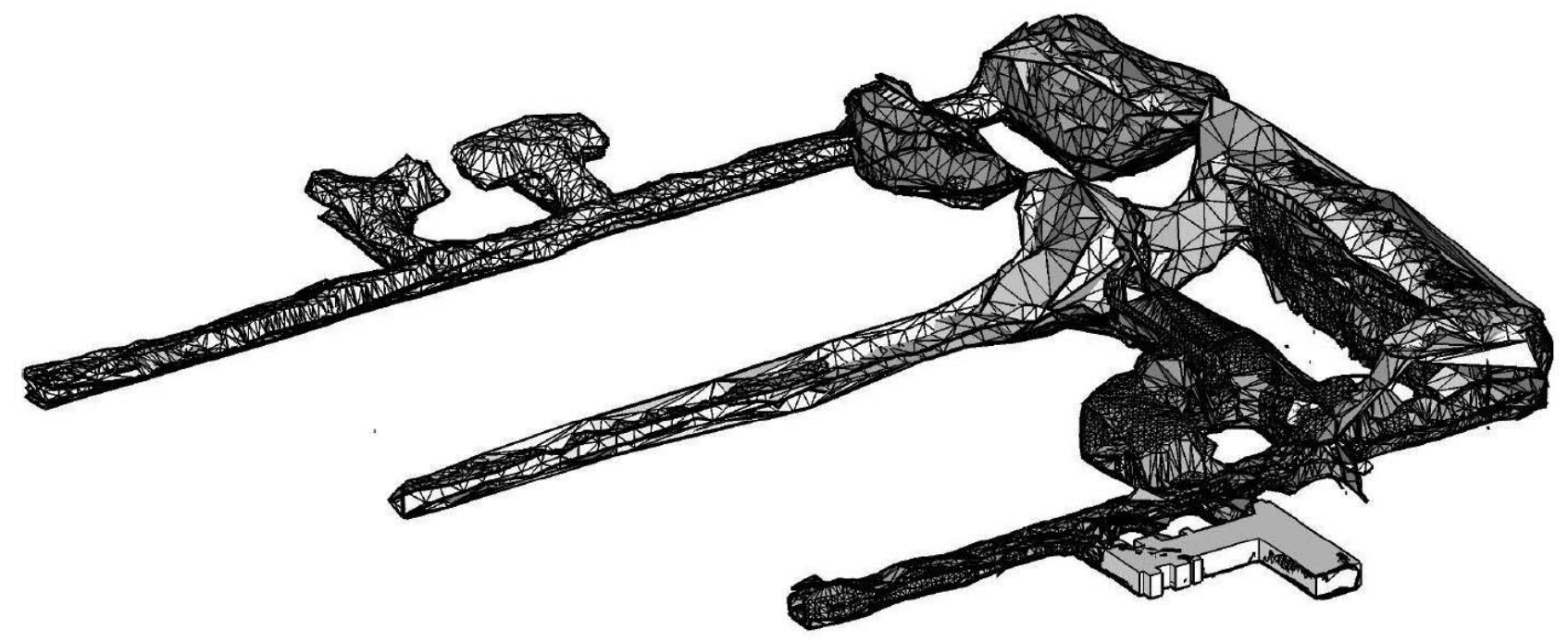

Fig. 9. The model of "Rzeczka" generated with the use of Undet for SketchUp plug-in.

\section{Conclusions}

The foregoing layouts, that present the areas of the Riese complex, were created with the use of classical methods, i.e. tacheometric surveying and levelling surveying, which enabled the creation of various plans of the objects, projections or maps. The terrestrial data extraction, done with the terrestrial laser scanning method, allowed for creating the numerical representation of "Rzeczka" in the form of threedimensional model which presents the geometry of the whole area in detail. The created model enables further processing for the purpose of conducting subsequent analyses regarding the construction or dimensions of the complex as well as creating derivative products on the model's basis, i.e. maps or profiles.

The three-dimensional reconstruction of the surface can be done in multiple graphical environments - open source as well as commercial type. The MeshLab program enables creating the reconstruction in a highly effective manner - due to the implemented algorithms, the surface reconstruction process has been done with a huge precision in a short period of time. The reconstruction of the surface that has complex geometry in the Undet for Sketchup programme is a very timeconsuming process and the generated TIN mesh does not present the geometrical details. In the case of generating models of regular shapes, e.g. guardroom located at the undergrounds of "Rzeczka" object, it needs to be noted that the Undet program is good with recognising surfaces of noncomplex geometrical shapes.

The model created in the MeshLab environment, due to the possibility of further processing and capability of exporting it in various formats, can also be used as a promotion tool, e.g. in a traditional way (various types of printouts) or in a digital way as an animation or interactive model available on the website.

This work was financed by the Polish Statutory Research Grant no. 0402/0137/16 and Grant no. 0401/0128/17.

\section{References}

1. G.L. Heritage and A.R.G. Large, Laser scanning for the Environmental Sciences (Wiley-Blackwell, 2009)

2. P. Kruszyński, Podziemia w Górach Sowich i Zamku Książ (Muzeum Gross-Rosen, Wałbrzych, 2004)

3. J. Rostowski, Podziemia III Rzeszy. Tajemnice Książa, Wałbrzycha i Szczawna-Zdroju (Dom wydawniczy Rebis, Poznań, 2010)

4. R. Staiger, Terrestrial Laser Scanning Technology, Systems and Applications (2nd FIG Regional Conference, Marakkech, 2003)

5. CloudCompare, official website www.cloudcompare.org, access date 22.10.2017

6. MeshLab, official website http://meshlab. sourceforge.net/, access date 22.10.2017

7. W.E. Lorensen and H.E. Cline, Computer Graphics, XXI(4), 163-169 (1987)

8. C. Montani, R. Scateni, R. Scopigno, The Visual Computer, 10 (6), 353-355 (1994)

9. F. Bernardini, J. Mittleman, H. Rushmeier, C. Silva, G. Taubin, IEEE Transactions on Visualization and Computer Graphics, 5 (1999)

10. M. Kazhdan, M. Bolitho, H. Hoppe, [In:] Symposium on Geometry Processing, Alla Sheffer and Konrad Polthier (Editors), The Eurographics Association, 61-70, DOI: 10.2312/SGP/SGP06/061070 (2006)

11. M. Bolitho, M. Kazhdan, R. Burns, H. Hoppe, [In:] Symposium on Geometry Processing, 69-78 (2007)

12. M. Bolitho, M. Kazhdan, R. Burns, H. Hoppe, [In:] Advances in Visual Computing, Lecture Notes in Computer Science Volume 5875, Springer, 678-689, DOI: 10.1007/978-3-642-10331-5 63 (2009) 
13. M. Kazhdan and H. Hoppe, ACM Transactions on Graphics, Vol. 32, No. 3, DOI: http://dx.doi.org/ 10.1145/2487228.2487237 (2013)

14. Undet for SketchUp, official website www.undet.com, access date 22.10.2017

15. F. Remondino, International Archives of Photogrammetry, Remote Sensing and Spatial Information Sciences, Vol. XXXIV-5/W10 (2003) 